CARNETS DE Carnets de géographes

GÉOGRAPHES.

4 | 2012

Géographies critiques

\title{
Géographies critiques » à la française »?
}

\section{Marianne Morange et Yann Calberac}

\section{(2) OpenEdition}

Journals

Édition électronique

URL : http://journals.openedition.org/cdg/976

DOI : $10.4000 /$ cdg. 976

ISSN : 2107-7266

Éditeur

UMR 245 - CESSMA

Référence électronique

Marianne Morange et Yann Calberac, « Géographies critiques » à la française »? », Carnets de géographes [En ligne], 4 | 2012, mis en ligne le 01 septembre 2012, consulté le 23 septembre 2020. URL : http://journals.openedition.org/cdg/976 ; DOI : https://doi.org/10.4000/cdg.976

\section{(c) (1) ()}

La revue Carnets de géographes est mise à disposition selon les termes de la Licence Creative Commons Attribution - Pas d'Utilisation Commerciale - Pas de Modification 4.0 International. 


\title{
GEOGRAPHIES CRITIQUES «A LA FRANÇAISE » ?
}

\author{
Marianne Morange \\ Université Paris Diderot \\ Géographe et urbaniste, SEDET, EA 4534 \\ marianne.morange@paris-diderot.fr \\ Yann Calbérac \\ Université Paris-Sorbonne (IUFM de Paris) \\ Géographe, UMR 8185 ENeC \\ yann.calberac@ens-lyon.org
}

\begin{abstract}
« À l'urgence de l'engagement s'est substituée pour nous la priorité du jugement. Cette rupture avec la tradition intellectuelle de type sartrien, qu'avaient illustrée Les Temps modernes, est ce qui a paru, sur le moment, la nouveauté la plus remarquée du Débat. On a vite compris que le refus de l'engagement politique partisan n'était que le produit d'une volonté de responsabilité civique. Dans le pays des Lumières, la présence de l'intellectuel dans la vie publique est une tradition. Il ne s'agit nullement d'y renoncer, mais bien au contraire, de lui redonner son lustre en lui trouvant son actualité. »

Pierre Nora, « Vingt ans de débat», Le Débat, 2000, page 9, http://www.ledebat.gallimard.fr/
\end{abstract}

A l'occasion des vingt ans de la revue Le Débat, Pierre Nora revient en ces termes sur le projet éditorial de ses fondateurs. Il évoque leur méfiance envers les affiliations partisanes et leur tentative, à l'aube des années 1980, de renouer avec une forme d'implication politique en somme plus « dégagée » pour les chercheurs en sciences sociales et humaines convaincus de la disqualification du combat des intellectuels marxistes.

L'essor contemporain des approches critiques dans la géographie française peut être interrogé à la lumière de ce manifeste et de ce tournant anti-partisan au sens où elles n'émanent pas d'un collectif d'intellectuels clairement identifié, engagé dans une œuvre politique de transformation du monde et assumant une mission historique, comme ce fut le cas pour certains de leurs aînés. On pense par exemple aux géographes tiers-mondistes comme Jean Dresch (Dresch, 1979) ou André Prenant qui ont pris parti dans la lutte contre le colonialisme, parfois aux côtés du Parti Communiste Français, ou encore à l'engagement d'Yves Lacoste et à la revue Hérodote dans les années 1970 (Lacoste, 1976) ${ }^{1}$.

\footnotetext{
${ }^{1}$ Voir le compte-rendu dans la rubrique « carnets de lecture ».
} 
Critiques mais pas nécessairement radicales ${ }^{2}$. Les géographies critiques contemporaines, en France, se caractérisent par un net pluralisme idéologique et un foisonnement d'approches méthodologiques et théoriques. Elles sont le produit d'influences variées, ce qui n'exclut pas la reconnaissance de certains héritages politiques. Elles s'organisent autour de thématiques, d'aires culturelles, de sous-champs disciplinaires, sans revendiquer le label critique. Elles restent donc assez cloisonnées les unes par rapport aux autres et ne disposent d'aucun support éditorial propre, alors qu'une telle tribune existe dans l'environnement anglophone avec la revue $A C M E^{3}$ dont le projet éditorial vise à réunir sous la bannière critique un grand nombre d'approches : " anarchisme, anti-racisme, environnementalisme, féminisme, marxisme, postcolonialité, poststructuralisme, études 'queer', situationnisme ou socialisme ».

Partant de ce constat, ce numéro des Carnets de géographes se propose d'offrir un espace de décloisonnement des approches critiques dans la géographie française. L'objectif est triple : en confrontant des géographies qui a priori échangent peu entre elles, il s'agit d'illustrer la contribution des géographes qui travaillent dans le contexte français au renouveau des pensées critiques, de rechercher les éventuelles spécificités d'une pensée critique «à la française » en géographie et de redonner quelque audience à la géographie, en tant que discipline, dans un champ où elle fait pour l'heure figure de parent pauvre.

C'est dans cet esprit qu'a été diffusé notre appel à articles. S'il était thématiquement ouvert, il s'adressait aux géographes francophones. Nous souhaitions ouvrir une arène expérimentale pour permettre à des géographes qui travaillent et élaborent leur pensée en langue française de s'exprimer sur la place de la critique dans la géographie qu'ils fréquentent et contribuent à bâtir. Nous n'avons toutefois reçu que des contributions émanant de chercheurs travaillant en France. Ce numéro porte donc sur les géographies critiques " à la française », au sens où elles sont produites dans le contexte institutionnel et académique français ${ }^{4}$. Nous avions en outre donné un sens assez large au terme «critique » en déclinant l'expression au pluriel et en évoquant des «approches construites autour d'une remise en question des catégories analytiques classiques (...), articulées à une volonté de peser sur les évolutions politiques et sociales. Cela afin de ne pas restreindre thématiquement les propositions et afin d'ouvrir ce numéro à toutes les manières de «faire avec », mais aussi, pourquoi pas, de «faire sans » la critique.

Les contours de la démarche critique sont en effet suffisamment flous pour que cette dernière soit parfois considérée comme une auberge espagnole théorique, scientifiquement inopérante. L'on trouve des traces de cette prévention aussi bien chez les partisans de la neutralité scientifique que chez les tenants d'une approche (plus) radicale. C'est par exemple le point de vue des organisateurs d'un prochain colloque consacré à « l'espace et aux rapports sociaux de

\footnotetext{
${ }^{2}$ Sur la distinction entre critique et radical, on peut se référer au texte de Cécile Gintrac dans le présent numéro et admettre avec elle que les approches radicales font partie des pensées critiques dont elles constituent une forme particulière du fait de leur rapport étroit à l'engagement militant et du fait de leur goût pour les " grands récits » et les analyses systémiques.

${ }^{3} \mathrm{http}: / /$ www.acme-journal.org/french.html.

${ }^{4}$ Le projet initial était ouvert aux autres disciplines et à toutes les sciences sociales qui traitent des questions spatiales. Nous n'avons cependant reçu que des contributions de géographes. Nous assumons donc dans cet éditorial la spécialisation disciplinaire du numéro qui en résulte.
} 
domination » dont nous rendons compte ici (voir entretien infra). Nous n'avons cependant pas reçu de proposition d'article dénonçant les limites ou les faiblesses des approches critiques. Ce numéro réunit donc des textes de géographes qui se revendiquent, chacun.e à leur manière, d'une telle approche. Nous avons voulu les laisser libres de la manière dont ils investiraient cet espace et aborderaient ce débat, entre enjeux théoriques et enjeux de méthode, ou encore en référence ou non au champ anglophone et aux critical (et radical) geographies.

\section{Besoins critiques?}

La géographie française n'a eu de cesse de questionner son rôle social et son rapport au politique (Calbérac et Delage, 2010). Elle n'a eu de cesse de «s'engager » dans le monde social. Dans ce numéro, Nicolas Bautès et Clément Marie dit Chirot soulignent l'influence des travaux menés dans les années 1960 par Renée Rochefort (Rochefort, 1961) sur la géographie sociale qu'ils pratiquent aujourd'hui ${ }^{5}$. Cependant, en géographie comme dans les autres sciences sociales, la pertinence de l'engagement critique a été sapée par la montée de l'impératif de «neutralité axiologique». Un soupçon pseudo-wébérien a grandi quant à la légitimité scientifique des approches dites non neutres ou fondées sur des jugements de valeur. On peut par exemple se souvenir de la charge de Jacques Brun (1994) contre les analyses de la ségrégation urbaine qui confondent selon lui dénoncer et comprendre, obscurcissent le phénomène plus qu'elles ne l'éclairent, et se révèlent au final peu heuristiques.

« Neutralité axiologique» au service de la juste compréhension des phénomènes observés. Cette traduction approximative de l'idée wébérienne de "Wertfreiheit» participe, selon Isabelle Kalinowski (2005), d'un détournement idéologique de la pensée de Max Weber. Ce glissement sémantique tardif aurait été opéré dans les années 1960 par les détracteurs de l'approche marxiste afin de récupérer politiquement la grande figure intellectuelle de Max Weber. Ce dernier n'opposait pas la Wertfreiheit (l'impératif moral de «non-imposition des valeurs » qui devrait selon lui guider toute démarche scientifique) à l'engagement politique. Il l'opposait à la propagande : le fait de s'appuyer sur une position académique dominante et sur une parole d'autorité pour imposer des valeurs en les présentant comme neutres. Traduire la Wertfreiheit par «neutralité axiologique " permettait de substituer à cette opposition subtile, celle, beaucoup plus simpliste, entre engagement et devoir de neutralité politique.

Dans les années 1980 et au début des années 1990, le succès de cette stratégie de détournement, signalé par l'avènement de l'ordre idéologique libéral (Cusset, 2006), aurait achevé de disqualifier scientifiquement et institutionnellement la figure du chercheur engagé et de discréditer la pensée critique en sciences sociales. Elle ne fut certes jamais totalement éclipsée si l'on pense par exemple au succès professionnel d'un Pierre Bourdieu et à l'audience de l'école de sociologie critique de l'EHESS durant cette période. Mais elle fut réduite à des niches académiques alors qu'elle avait été dominante dans les décennies précédentes.

Redécouvrir, ressusciter, mais aussi refonder la pensée critique autour de nouvelles approches théoriques, dans une relation reconfigurée et souvent plus distante à l'engagement politique.

\footnotetext{
${ }^{5}$ Voir aussi dans ce numéro, l'entretien réalisé avec Bernard Bret.
} 
C'est le défi que s'efforcent de relever ce que Razmig Keucheyan (2010) a baptisé les «nouvelles pensées critiques ». La vitalité, voire l'effet de mode qui entoure ces approches, relève d'un désir de rupture avec la parenthèse des années 1980-1990 et d'une aspiration à s'affranchir des injonctions à la neutralité axiologique qui l'ont caractérisée. Leur succès serait donc lié à un moment de rupture et de crise idéologique et institutionnelle.

De manière plus anecdotique, cette crise renvoie parfois à un besoin de résistance contre les transformations récentes de l'Université française. Mise en demeure par des pouvoirs publics acquis au tournant néolibéral de la stratégie de Lisbonne, de devenir (enfin) " utile » (professionnalisante, évaluatrice de « compétences » et productrice d'une recherche appliquée, soumise au dictat du temps atrophié de l'appel à projets et mise au service des grandes entreprises privées ou publiques), elle se trouve sommée de redéfinir sa fonction sociale dans le cadre d'une autonomie budgétaire de type entrepreneuriale. La pensée critique s'en trouve marginalisée (quand elle n'est pas jugée suspecte a priori pour des raisons idéologiques) et la recherche fondamentale, condamnée à la portion congrue, que ce soit en lettres et en sciences humaines et sociales ou dans les sciences dites dures ${ }^{6}$.

\section{Trois chemins critiques}

Depuis le milieu des années 1990, la pensée critique en géographie, comme dans nombre de disciplines, renait donc sous des formes multiples. On peut penser aux travaux sur les relations entre éthique et géographie menés au sein du laboratoire ESO et ancrés dans une vigoureuse tradition de recherche en géographie sociale (voir dans ce numéro, la contribution de Nicolas Bautès et Clément Marie dit Chirot), ou aux réflexions menées sur ce même thème par des chercheurs plus isolés (voir par exemple Morelle et Ripoll, 2009 ou Collignon 2010). On peut, parmi les initiatives très récentes, évoquer la création de la revue Justice spatiale |Spatial Justice qui questionne les relations entre espace et justice, les travaux d'Arnaud Brennetot sur les manières dont la géographie mobilise la notion de justice (Brennetot 2009, 2011), ou encore l'organisation du colloque sur «Espaces et rapports sociaux de domination: chantiers de recherche » en septembre 2012 à l'Université de Paris Est Marne-la-Vallée? .

Il en résulte une grande diversité de points de vue et de manière de faire ou d'être critique. Les contributions que nous avons reçues émanent donc de domaines géographiques très divers et abordent des thématiques très variées : gentrification, services urbains, renouvellement urbain/ville créative, grands projets d'aménagement portuaire/aménagement régional, spéculation foncière et immobilière liée au tourisme, mais aussi littéracie, géographie des mobilités et épistémologie ${ }^{8}$. Elles portent sur des terrains situés aussi bien au Nord (Bruxelles,

\footnotetext{
${ }^{6}$ De ce point de vue, Les Carnets de géographes nous ont paru constituer un support approprié pour ce numéro. En tant qu'espace d'expression libre de tout référencement, ils peuvent accueillir des textes au format parfois difficiles à diffuser dans les revues établies et la diversité de leurs rubriques permet d'interroger la construction des savoirs et les formes d'engagement des auteurs.

${ }^{7}$ Voir l'entretien réalisé avec les organisateurs du colloque pour le présent numéro et retranscrit ici.

${ }^{8}$ Même si le Sud est plus représenté et les études urbaines, mieux servies. Cécile Gintrac, Martine Drozdz et Sarah Mekdjian constatent également que les études urbaines ont dominé les rencontres du réseau international des géographes critiques à Francfort en 2011 (voir compte-rendu dans ce numéro).
} 
Montréal, Paris) qu'au Sud (Tanger, villes du golfe, Mali, Le Cap, Maputo, Inde, Mexique et Brésil)

On peut néanmoins les regrouper en trois types d'approche :

\section{1-L'approche théorique : la critique comme grille de lecture du monde}

Quatre textes mobilisent ou élaborent un cadre théorique ou une grille de lecture critiques pour pénétrer un phénomène spatial : Sabine Planel propose une approche théorique très originale de la notion «d'espace juste», appliquée au cas du port de Tanger Med, qui combine les apports de la critique foucaldienne sur le pouvoir et ceux des geographies of scales anglophones (géographie des échelles) ; Anne Clerval et Mathieu Van Criekingen explicitent, sous la forme d'un dialogue scientifique, la manière dont ils mobilisent dans leurs travaux respectifs sur Paris et sur Montréal et Bruxelles, la notion de gentrification, forgée au Royaume-Uni et d'abord travaillée dans le monde anglophone; Frédéric Barbe propose une «géographie critique appliquée à la culture » (la littéracie) afin de dénoncer «la force du système «transcolonial» qui a fait échouer l'utopie scolaire de l'Indépendance » au Mali, ainsi que les "obstacles à la formation d'un marché national de la littéracie cohérent»; il dessine les contours d'une «action publique plus soucieuse de ménager le territoire et la société en favorisant un accès universel à la lecture-écriture ». Roman Stadnicki et Manuel Benchetrit sillonnent les villes du golfe et mettent en image les pratiques de leurs citadins ordinaires afin de nuancer la lecture catastrophiste qu'a fait de ces villes l'anthropologie radicale anglophone de Mike Davies et Daniel Monk, selon eux abusivement généralisante et hors-sol. S'ils se proposent de « dépasser la critique », leur proximité avec le terrain, leur souci de la complexité des dynamiques socio-spatiales et de la tension entre spécificités et montée en généralité les rapprochent de la Nouvelle critique sociale.

\section{2- L'approche épistémologique : la pensée critique comme objet ou question de recherche}

Deux auteurs constituent la géographie radicale anglophone en objet de recherche: Cécile Gintrac étudie la construction de ce champ et questionne les relations entre pensées critique et radicale, dans un aller-retour entre la France et les Etats-Unis ; Camille Vergnaud esquisse les contours de « la figure de l'enseignant-chercheur-activiste » et analyse ses « concrétisations » dans le contexte états-unien. Elle en décrit trois incarnations : la construction d'un espace «d'academivism » à l'université de Syracuse (par la mise à disposition des apports de la technologie du SIG aux « communautés »), «l'empowerment» des « communautés » par des « chercheurs facilitateurs », et « la géographie radicale de bibliothèque » de Don Mitchell qui met plus classiquement son savoir au service des groupes dominés pour les aider à fourbir leur argumentaire militant et à adapter leurs moyens d'action.

Par ailleurs, Nicolas Bautès et Clément Marie dit Chirot proposent un manifeste «pour une géographie sociale de l'action » qui dépasse le simple objectif de dévoilement pour prendre les chemins d'une géographie "performative ». Ils mobilisent pour ce faire leurs expériences de terrain respectives sur la spéculation foncière et immobilière dans une ville touristique du 
Mexique et sur les expulsions dans le cadre du « renouvellement urbain » à Rio de Janeiro et Mumbai.

\section{3- L'approche réflexive : la critique comme méthode}

Trois textes enfin proposent un travail réflexif sur les pratiques et les savoirs de leurs auteurs. Ces trois contributions nous semblent particulièrement précieuses sur le plan disciplinaire car, comme le soulignent Nicolas Bautès et Clément Marie dit Chirot, la géographie, contrairement à d'autres sciences sociales, fait souvent l'économie de ces explicitations (parfois pour des raisons de format et de contraintes éditoriales) :

Karine Ginisty revient sur les difficultés qu'elle a rencontrées pour penser la justice spatiale à Maputo dans l'accès à l'eau potable et sur le travail de déconstruction-reconstruction de cette notion que le terrain lui a imposé de mener ; Thomas Radovcic explique comment il a progressé dans sa compréhension des injustices au Cap en s'affranchissant d'une grille de lecture post-apartheid strictement socio-raciale pour mobiliser la littérature anglophone sur la néolibéralisation et la gouvernance urbaine ; Nathalie Bernardie-Tahir et Camille Schmoll lèvent le voile sur les coulisses de leur expérience de terrain dans les camps de migrants dits « irréguliers » à Malte ; elles dissèquent le «mouvement de balancier constant entre voies de traverse militantes et travail académique, entre pas de côté, mouvements de décentrement et de recentrage, jeux de distance et d'engagement, processus d'objectivation et de subjectivation » qu'il leur semble urgent d'expliciter pour tenir la tension entre engagement et neutralité scientifique.

\section{Convergences critiques}

Au-delà de leur diversité et de leurs divergences, ces géographies critiques partagent trois caractéristiques qui sont liées à certaines spécificités disciplinaires : elles assument une forme de méfiance par rapport aux ambitions théoriques trop globalisantes qu'elles renvoient à la primauté du terrain ; elles manifestent une certaine distance vis-à-vis de l'engagement politique, envisagé fondamentalement lui aussi dans la relation à la complexité du terrain; elles témoignent d'une capacité à combiner une approche critique moderniste et certains apports des théories post-modernes. De ce fait, les géographies critiques «à la française» entretiennent un rapport ambigu aux géographies critiques anglophones.

\section{Pensées critiques ou théories critiques?}

Le débat sémantique et épistémologique sur les sens et les pratiques de la critique en sciences humaines et sociales a déjà été largement engagé (voir le numéro que la revue Tracés lui a consacré en 2007, en particulier l'éditorial d'Arnaud Fossier et Anthony Manicki qui propose une synthèse sur la question). Or la géographie est absente de ce numéro, consacré à la sociologie, à la philosophie, à l'histoire et aux études littéraires. De même, elle est à peine évoquée dans les deux ouvrages de référence, pourtant très riches sur les plans thématique et disciplinaire, qui ont récemment entrepris de baliser le champ des pensées critiques: la 
« cartographie » que Razmig Keucheyan (2010) met à notre disposition pour nous aider à arpenter ce dédale théorique et idéologique et, sous une forme plus militante, « l'instantané au moins partiel de cette constellation » éclectique que nous offre un collectif d'auteurs (2011). Ses apports y sont réduits à la géographie radicale, et plus spécifiquement à l'œuvre de David Harvey, seul jugé digne d'entrer au panthéon des pensées critiques contemporaines du fait de la portée de son système théorique ${ }^{9}$. Nicolas Bautès et Clément Marie dit Chirot voient dans son extrême popularité une forme de «normalisation » et un risque d'appauvrissement de la pensée critique en géographie.

A cette exception près, la géographie occuperait une place marginale dans le grand mouvement de refondation des «théories » critiques. Cette grande absence reflète sans doute la marginalité disciplinaire de la géographie dans les sciences sociales. L'audience de David Harvey s'est d'ailleurs largement formée en dehors de la géographie et il est sans doute le géographe le plus cité dans les autres sciences sociales. Le caractère faiblement audible des géographes critiques produites en France est aussi lié au fait qu'elles sont dispersées et émanent de chercheurs qui ne se réfèrent pas à de grandes figures tutélaires, voire qui manifestent une certaine défiance à l'égard de leurs systèmes théoriques cohérents et fermés. Comme en témoigne ce numéro, ces géographies sont largement affranchies des ambitions de la « totalisation historique hégélienne » (Cusset et Keucheyan, 2010) qui caractérise leur alter ego anglophone. De ce point de vue, la part d'apport théorique jugée nécessaire pour jouir du label « critique » reste floue : Razmig Keucheyan par exemple parle tantôt de "pensées », tantôt de "théories » critiques mais il privilégie les architectures théoriques cohérentes et fermées, également très prisées dans le monde anglophone.

La force de la tradition empirique dans la géographie française a été peu propice à l'élaboration de «théories critiques». Elle a poussé à subordonner l'abstraction théorique aux enseignements du terrain (Calbérac, 2010; Volvey, Calbérac et Houssay-Holzschuch, dir., $2012)^{10}$. La théorie n'est pas ignorée mais elle n'est pas convoquée a priori pour être testée sur un terrain presque quelconque comme c'est souvent le cas dans les géographies anglophones qui font la part belle aux longs états de l'art théoriques. Le texte de Karine Ginisty est emblématique de la manière dont les géographes critiques pensent la tension entre terrain et théorisation : elle expose la difficulté à articuler les apports de la philosophie politique et de la philosophie morale sur la notion de justice à la réalité sociale et politique de Maputo, où la force du contrôle social exercé par le parti hégémonique entrave l'émergence de la contestation politique et d'un sentiment d'injustice. «L'enquête en littéracie malienne » de Frédéric Barbe repose, de même, sur une patiente accumulation de données historiques, sociales, politiques qui démontre la complexité des processus de domination «transcoloniaux » à l'œuvre (concurrence éditoriale injuste, difficultés de la scolarisation et débat politique sur les langues « nationales » ...).

\footnotetext{
${ }^{9}$ Henri Lefebvre est également cité par Razmig Keucheyan, mais de manière incidente et du côté des héritages aujourd'hui remobilisés pour son idée du « droit à la ville » et sa conceptualisation de l'espace «produit ».

${ }^{10}$ Voir le compte rendu de lecture dans ce numéro des deux numéros que la revue Hérodote a consacrés en 1977 et 1978 à la question du terrain.
} 
Trois textes assument néanmoins un rapport plus intime à la théorisation. Le texte co-signé par Anne Clerval et Mathieu Van Criekingen et l'article de Thomas Radovcic s'inscrivent d'ailleurs dans un dialogue direct et explicite avec la littérature anglophone. Mais même là, le terrain reste premier dans la démarche: si Anne Clerval trouve séduisante la tentative de totalisation théorique de David Harvey, elle explique que c'est d'abord l'étude de la transformation sociale des quartiers parisiens qui l'a menée à la notion de gentrification ; pour Thomas Radovcic, c'est la rencontre avec le réseau d'élites du Cap qui l'a conduit à s'interroger sur la gouvernance et l'exercice du pouvoir dans le contexte néolibéral et entrepreneurial et à dépasser une grille de lecture a priori raciale. Sabine Planel propose le texte le plus théorisant. Il reprend d'ailleurs le canon des articles anglophone : la première partie, dédiée au cadre théorique, est suivie d'une étude de cas. Mais même dans ce cas, la prudence l'emporte en conclusion quant à la portée de «l'hypothèse » théorique testée ici.

\section{La place du projet de transformation sociale}

Dans notre appel à articles, nous avions postulé, pour la pensée critique, cette double exigence : en tant que science sociale, la géographie dispose de (ou est à même d'élaborer) l'appareil théorique permettant de rendre intelligibles les formes de domination et d'exploitation liées à l'espace et au territoire, ainsi que les injustices spatiales; elle doit (au sens d'un devoir éthique) le mobiliser pour faire œuvre émancipatrice et participer à la transformation de la société afin de la rendre (plus) juste.

Ce double impératif nous dégage d'une approche strictement kantienne qui confine la critique dans «l'examen évaluatif des catégories et des formes de connaissance afin d'en déterminer la validité et la valeur cognitives » (Wacquant 2001). Si l'on admet que les pensées critiques mêlent nécessairement « le descriptif » et « le normatif » (Keucheyan, 2010), elles doivent se situer dans l'engagement, ou du moins par rapport à un objectif de transformation sociale et d'émancipation politique. La critique en géographie ne relèverait ainsi pas seulement d'un acte d'affranchissement et de subversion envers une parole d'autorité grâce au travail de la raison critique (au sens kantien) ${ }^{11}$, même si cette dimension peut être présente.

Cette tension entre examen critique et prescription politique est proche de la position des éditeurs de la revue $A C M E$, précédemment citée, pour qui «l'analyse critique et radicale est perçue comme faisant partie d'une pratique sociale et politique dont le but est de défier, transformer ou démanteler les relations, systèmes et structures dominants du capitalisme, ainsi que les régimes d'exploitation, oppression, impérialisme, néo-libéralisme, agression nationale et destruction environnementale qui les accompagnent». Elle nous rapproche, toujours selon Loïc Wacquant, d'une perspective marxienne qui « se donne pour tâche de porter au jour les formes cachées de domination et d'exploitation (...) afin de faire apparaître, en négatif, les alternatives qu'elles obstruent et excluent ».

\footnotetext{
${ }^{11}$ Sur la distinction entre approche kantienne et marxienne et le caractère dualiste de la proposition de Loïc Wacquant, on peut se rapporter à l'article de Cécile Gintrac.
} 
Si l'on admet, avec Razmig Keucheyan, qu'une " théorie critique se pose nécessairement la question de son rapport à l'action », les formes "d'action» accessibles aux chercheurs critiques sont très variées. Entre diffusion et co-production de la connaissance avec les citoyens, par-delà le cercle académique, les interactions avec les exploités et les dominés peuvent aller de l'effort de conscientisation à la collaboration avec des groupes et organisations militantes. L'action peut viser un public estudiantin quand il s'agit d'élaborer des cours dédiés à la présentation des théories critiques afin de sensibiliser les étudiants aux questions de domination. Elle peut viser l'ensemble de la communauté scientifique quand il s'agit d'affiner des appareillages théoriques dans un dialogue plus interne à l'académie.

Or les géographes critiques qui s'expriment ici sont assez en retrait par rapport à la nécessité de l'engagement politique dans les sociétés étudiées. Ils ne l'envisagent pas comme constitutif ou fondateur dans leur approche. Si certains évoquent des expériences individuelles de militantisme dans le cadre de leurs recherches, ils ne construisent pas leur démarche scientifique dans ce cadre, ni en référence première à elles. Au demeurant, aucun d'eux ne se réclame d'un collectif scientifique et académique militant. Leur engagement est indirect ou ponctuel, souvent situé aux frontières de la pratique académique. Il résulte d'un mouvement centrifuge qui part de l'université pour aller vers le monde politique et la lutte sociale, et jamais l'inverse.

Frédéric Barbe par exemple pratique une critique kantienne au sens où il déconstruit « une croyance littéraire fortement indurée dans la grande culture française [qui] tend à réduire le champ légitime de l'écriture et de la lecture à la 'littérature' et plus encore à son 'canon', soit une part infime du corpus écrit disponible»; il propose à partir de ce travail de glisser vers une « recherche-action » sous la forme d'un «atelier/compte-rendu d'enquête » et formule des propositions concrètes de création d'un fonds public de financement du livre au Mali. La difficulté à «s'engager» est accrue pour les chercheurs qui travaillent sur des sociétés extérieures à celle dans laquelle ils vivent (Brésil, Mexique, Inde, Afrique du Sud, Mozambique, Malte...). Ce qui nous renvoie à la question du positionnement dedans/dehors, notamment dans le contexte post-colonial, et à la possibilité même d'une posture critique en ce qui concerne les travaux sur « les Suds » menés par les chercheurs vivant aux « Nords ».

Trois textes se distinguent par leur désir de questionner directement la relation à l'engagement. Nicolas Bautès et Clément Marie dit Chirot y consacrent leur article. Ils parlent «d'engagement critique » et se réclament d'une " géographie sociale de l'action ", questionnant les « limites actuelles de la diffusion des savoirs académiques que construisent les sciences sociales ». Plus réservées sur leur rapport à l'engagement militant, Nathalie Bernardie-Tahir et Camille Schmoll expliquent comment elles ont dû affronter et assumer seules le dilemme moral sur la nécessité de « faire quelque chose », de " dénoncer », " sans en rabattre sur ses exigences scientifiques», dans la situation complexe et tendue qu'elles découvraient, et comment elles ont tenté de ne pas «mélanger les registres », sans réellement y parvenir de manière totalement satisfaisante. Camille Vergnaud pratique une forme d'egogéographie (Lévy, 1995) qui lui permet d'expliquer son travail sur les géographies critiques 
anglophones par rapport à ses doutes et ses difficultés personnels par rapport à l'engagement dans une carrière académique.

Par ailleurs, l'engagement, quels que soient son degré et sa nature, se pense avant tout dans la relation au « terrain ». Pour Nicolas Bautès et Clément Marie dit Chirot, il résulte en partie du contact avec les collectifs de défense des victimes de la spéculation immobilière et du renouvellement urbain. Les expulsés notamment sont plus accessibles et prêts à dialoguer avec un universitaire que les représentants des expulseurs, ce qui place les chercheurs immédiatement du côté des opprimés. C'est d'ailleurs tout le sens de l'engagement «à rebours », évoqué dans cet article, de Monique et Michel Pinçon-Charlot qui travaillent sur la grande bourgeoisie ${ }^{12}$. Nathalie Bernardie-Tahir et Camille Schmoll quant à elles soulignent les difficultés auxquelles elles se sont trouvées brutalement confrontées quand elles ont découvert « le terrain », c'est-à-dire l'inhumanité de la situation dans laquelle vivent les migrants à Malte et le « choc » qu'elles ont subi, en partie précisément du fait de leur absence de préparation politique, et malgré leur connaissance fine des questions migratoires. C'est le terrain qui mène à l'engagement et non l'inverse.

L'indépendance idéologique de ces géographies critiques enfin se lit notamment dans leur autonomie vis-à-vis de la géographie marxiste française des années 1960-70 qu'elles ne convoquent quasiment pas. Le regain d'intérêt pour les pensées critiques semble devoir davantage à la permanence de la figure historiquement construite de l'intellectuel engagé (Ory et Sirinelli, 1986) et indépendant : le «jugement », certes souvent élaboré au sein d'un collectif scientifique (le laboratoire ou le réseau de recherche) ou dans un contexte intellectuel favorable, mais pas «l'engagement» dans un groupe de chercheurs militants et proches des réseaux activistes et des enjeux politiques immédiats, pour reprendre la distinction de Pierre Nora.

\section{Le rapport au dévoilement et à la post-modernité}

Les géographes qui s'expriment ici manifestent des ambitions de dévoilement propres à la pensée critique moderniste, que l'on se réfère au projet de l'école de Francfort d'une pensée critique qui éclaire dans le but d'altérer l'ordre social et politique (Wiggerhaus, 1993) ou à la sociologie critique d'un Bourdieu qui oppose le «sens savant» au "sens commun». Mais presque tous sont sensibles à certains apports de la post-modernité :

Sabine Planel dévoile des mécanismes de domination scalaire en mobilisant à la fois la filiation lefebvrienne (sur les modalités de "production de l'espace » juste) et la conception fluide des dynamiques scalaires de Michel Foucault. Egalement à la croisée entre pensée critique moderniste et post-moderne, Frédéric Barbe propose un manifeste pour une littéracie dégagée des formes de domination «transcoloniales » au Mali, dans la lignée des subaltern studies, autant qu'il dénonce " 'l'ajustement structurel de la littéracie' (sa déstructuration sous l'effet de la marchandisation imposée) » et prône « la constitution d'un marché de la littéracie plus

\footnotetext{
${ }^{12}$ Voir à ce sujet le compte-rendu de lecture proposé dans la section « carnets de lectures » : Delphine Naudier et Maud Simonet (dir.), 2011, Des sociologues sans qualités ? Pratiques de recherche et engagements, Paris, La Découverte.
} 
«juste », fondé sur un marché de l'édition moins inégalitaire et sur une refonte des politiques linguistiques. Anne Clerval et Mathieu Van Criekingen soulignent l'importance de la lecture néomarxiste de Neil Smith dans leur analyse de la gentrification mais évoquent aussi les apports de "l'intersectionnalité » dans la compréhension de ce phénomène et la nécessité d'enrichir l'approche strictement classiste par une approche de genre, de race... (même s'ils ne développent pas cet aspect ici). Dans une certaine mesure, le passage d'une «sociologie du dévoilement » à une sociologie "performative» pour Nicolas Bautès et Clément Marie dit Chirot intègre aussi un souci de la subalternité, par exemple dans la référence à «une expérience de coproduction des savoirs géographiques sur les formes d'autonomie développées par des organisations sociales indigènes de différentes régions mexicaines ».

Certains textes sont plus explicitement et directement sensibles aux apports du postmodernisme sur la question du positionnement et de la subjectivité du chercheur, et plus conformes aux injonctions de la « sociologie de la critique » (Boltanski, 1990), soucieuse de ne pas substituer une parole critique savante à la parole des victimes et des exploités. Camille Schmoll et Nathalie Bernardie-Tahir « donnent la parole » aux migrants dits irréguliers à Malte, afin de «montrer leur capacité de résistance et d'autonomisation en mettant en évidence les multiples formes de tactiques et de stratégies, individuelles et collectives, mises en place pour améliorer leur situation au quotidien et, plus largement, en leur restituant la possibilité d'être narrateurs de leur propre histoire ». Deux textes insistent, de même, sur les dangers d'une interprétation surplombante et externe des situations de domination au seul prisme d'une lecture marxiste inspirée de la géographie radicale anglophone (Roman Stadnicki), ou au seul prisme des théories de la justice produites par la philosophie européenne et nord-américaine (Karine Ginisty). Ils insistent tous deux sur la nécessité pour la pensée critique d'émaner du « terrain » (pour parler en géographe) et de se construire par rapport aux situations sociales, politiques et culturelles concrètes qu'elle rencontre plus qu'en fonction de lectures théoriques a priori et closes émanant de chercheurs en situation de «monopole» intellectuel (Walzer 1990). Cela implique un décentrage inconfortable et une remise en cause radicale de la situation de domination intellectuelle du chercheur que la géographie, dans son rapport au terrain, est particulièrement à même de mener.

Ce goût pour la «compréhension » explique sans doute en partie pourquoi les contributions réunies ici sont dominées sur le plan méthodologique par les approches qualitatives. Protocole d'entretiens semi-directifs pour Nathalie Bernardie-Tahir et Camille Schmoll ainsi que pour Karine Ginisty, «position d'observation participante» pour Frédéric Barbe, entretiens institutionnels pour Sabine Planel, Thomas Radovcic et Nicolas Bautès et Clément Marie dit Chirot, «géophotographie» pour Roman Stadnicki et Manuel Benchetrit... Ces méthodes sont par ailleurs parfois librement combinées. Nous n'avons pas reçu de proposition émanant de spécialistes des approches quantitatives et de l'analyse spatiale (seuls Anne Clerval et Mathieu Van Criekingen évoquent la mobilisation de données statistiques dans leur analyse de la gentrification, mais cet aspect n'est pas au centre de la discussion qu'ils proposent ici), ce qui laisse ouverte cette question : une certaine analyse spatiale caractérisée par sa tradition de recherche des lois (nécessairement objectives ?) de l'espace peinerait-elle davantage que les 
géographies fondées sur des méthodes qualitatives à assumer le potentiel critique de son analyse?

\section{Le rapport aux critical geographies}

Primauté du terrain, défiance par rapport aux théories trop globalisantes et prudence par rapport à l'engagement politique. C'est ce qui distingue les géographies critiques « à la française » des critical geographies et de la géographie radicale anglophone, davantage que des choix thématiques et des objets de recherche qu'elles ont souvent en partage (la justice spatiale, la néolibéralisation, le contrôle des migrations, la ségrégation, les injonctions à la mobilité, les discriminations de genre...). Cela implique des relations particulières à ces géographies critiques venues d'ailleurs, entre curiosité, attrait et distance critique.

Les critical geographies sont désormais bien connues en France où elles font l'objet d'une forte curiosité depuis les années 1990 et $2000^{13}$. Elles ont été diffusées en même temps que les textes des auteurs post-modernes venus des campus d'Amérique du Nord (Cusset, 2003). La géographie radicale anglophone en particulier bénéficie d'un fort engouement. Certains géographes français ont joué un rôle de passeur dans sa diffusion : on pense au travail séminal de B. Collignon (in Staszak, 2001) ou à la traduction totale ou partielle de plusieurs ouvrages de David Harvey depuis $2008^{14}$, récemment invités dans plusieurs écoles et universités. Cette géographie patiemment façonnée sur le plan institutionnel depuis les années 1960 (à travers la revue Antipode dont elle s'est dotée ${ }^{15}$ ) a «fait école» sans jamais s'essouffler ni renier sa filiation marxiste.

Les géographes français entretiennent des relations contrastées avec ces géographies venues d'ailleurs. Si la revue Justice spatiale | Spatial Justice a engagé un dialogue avec le monde anglophone autour des questions de justice et d'espace, elle échange pour l'instant davantage avec les études urbaines et les auteurs post-modernes qu'avec la géographie anglophone critique ou radicale; le dialogue et la filiation avec la géographie radicale incarnée par David Harvey sont en revanche recherchés et assumés par les organisateurs du colloque « Espaces et rapports sociaux de domination : chantiers de recherche », dont Anne Clerval qui a co-écrit dans ce numéro ${ }^{16}$. Dans ce numéro, les positionnements sont donc logiquement très variés :

- Trois textes bâtissent largement leur contribution en référence aux géographies anglophones, dans leur lignée ou en rupture avec elles. Anne Clerval et Mathieu Van Criekingen se réclament

\footnotetext{
${ }^{13}$ Dans l'entretien publié dans ce numéro, Bernard Bret explique qu'il ne prend connaissance des travaux de David Harvey qu'à la faveur de l'anthologie coordonnée par Jean-François Staszak (2001).

${ }^{14}$ En 2008 : Géographie de la domination, Paris, Les prairies ordinaires. En 2010 : Géographie et capital. Vers un matérialisme historico-géographique, Paris, Syllepse et Le Nouvel impérialisme, Paris, Les prairies ordinaires. En 2011 : Le capitalisme contre le droit à la ville. Néolibéralisme, urbanisation, résistances, Paris, Editions Amsterdam. En 2012: Paris, capitale de la modernité, Paris, Les prairies ordinaires. On peut souligner dans cette redécouverte de ces textes le rôle central des éditeurs qui renouent avec la figure de l'éditeur engagé des années 1960 aux années 1980 (Hage, 2010).

${ }^{15}$ Fondé dans le contexte des mouvements sociaux de 1968, elle a récemment pris une nouvelle ampleur en créant un site Internet plus interactif quoiqu'encore largement académique dans son format (http://antipodefoundation.org/).

${ }^{16}$ Voir l'article d'Anne Clerval et Mathieu Van Criekingen dans ce numéro.
} 
de l'approche radicale de David Harvey et Neil Smith qu'ils souhaitent transposer sur leurs terrains ; Roman Stadnicki et Manuel Benchetrit soulignent au contraire ses limites pour leurs terrains, à travers une critique de Mike Davies et Daniel Monk. On retrouve chez eux l'attachement à la tradition de recherche cultivée par les spécialistes des pays du Sud, d'une géographie inductive, sensible aux enseignements du terrain. Thomas Radovcic enfin mobilise l'article fondateur de Neil Brenner et Nik Theodore sur "l'urbanisation du néolibéralisme» dans sa lecture des injustices au Cap.

- Deux textes se situent dans un rapport de voisinage bienveillant et curieux à cette littérature, mais de manière plus tangente : Frédéric Barbe s'inspire des New Literacy Studies, empruntant le terme de littéracie au québécois ; Nicolas Bautès et Clément Marie dit Chirot reconnaissent que la «géographie sociale partage [...] avec la géographie radicale anglo-saxonne un certain nombre d'éléments théoriques, ainsi les références à Henri Lefebvre, au marxisme, aux classes sociales, etc. » mais cette géographie n'est pas centrale dans leur analyse. Sabine Planel la mobilise pour procéder à une hybridation théorique inédite entre pensée critique foucaldienne et geographies of scales.

- Deux textes enfin associent leur intérêt pour la pensée critique à une tout autre inspiration, et non aux apports d'un dialogue théorique franco-anglais: la fréquentation, notamment méthodologique, d'autres disciplines: l'ethnométhodologie et les subaltern studies pour Nathalie Bernardie-Tahir et Camille Schmoll. Pour Karine Ginisty, c'est la construction du « terrain » qui la rend attentive aux pièges de l'altérité, et sa familiarité avec l'anthropologie urbaine.

La proximité avec les géographies critiques anglophones semble résulter d'une combinaison complexe entre nature du terrain, champ thématique, et sensibilité personnelle des chercheurs. L'intérêt pour l'approche anglophone semble plus net parmi les spécialistes des questions urbaines. Il est peut-être lié à la notoriété de ces approches, et notamment de la géographie radicale, dans le champ des études urbaines. Leur succès les rend difficilement contournables, même s'il s'agit de les rejeter. En outre, même s'il ne s'agit pas d'une règle, c'est également le cas pour les terrains anglophones ou fréquentés par les géographes anglophone (Le Cap, les villes de la péninsule arabo-persique, ou les villes du Nord plus largement). Sur les terrains moins familiers aux géographies anglophones (Mali, Maroc, Mozambique), les chercheurs semblent un peu moins marqués par ces approches et les contournent parfois plus aisément.

C'est finalement surtout en matière d'engagement que les géographies critiques «à la française » manifestent un intérêt pour les efforts des géographes critiques anglophones qui débattent intensément des moyens d'action qui sont à leur disposition : plusieurs auteurs évoquent les expérimentations en matière de community-based research, d'action research, de participatory action-research (par exemple la fondation d'une «géographie populaire » par Don Mitchell). Ces efforts d'ouverture vers ce que l'on qualifierait sans doute de « société civile » ne constituent pas (encore ?) un attendu dans les carrières des enseignants-chercheurs 
en France ${ }^{17}$. Mais elles tendent à se développer. Elles intéressent donc tout particulièrement Nicolas Bautès et Clément Marie dit Chirot, Cécile Gintrac et Camille Vergnaud.

Leur tendance commune au désengagement politique, pourrait ainsi mener, par-delà la radicalité, à des convergences critiques. Comme le rappelle Cécile Gintrac, dans le monde anglophone, l'explosion des pensées critiques s'est accompagnée d'une dissolution partielle de la radicalité. Notons que dans les années 1960, le terme "radical " avait été retenu pour accueillir une plus grande diversité d'engagements et des formes de pensées dissidentes de la pensée marxiste, alors dominante. En somme, la pensée critique actuelle dans le monde anglophone serait à la radicalité ce que la radicalité a été, un temps, au marxisme.

Pour l'instant cependant, les convergences entre géographies critiques à la françaises et critical geographies semblent difficiles notamment du fait d'une forte dissymétrie institutionnelle. Les crititical geographies, quoique très hétérogènes (elles embrassent aussi bien les subaltern que les postcultural ou les postcolonial studies par exemple), constituent une nébuleuse clairement identifiée dans le monde anglophone. Elles se sont dotées de revues (comme $A C M E^{18}$ ), bénéficient de sessions spécifiques dans les colloques (une cinquantaine par exemple aux rencontres de 1'Association of American Geographers en 2012), de collections dédiées chez de grands éditeurs (par exemple aux presses de l'Université de Californie), d'articles dans des encyclopédies, de manuels (on pense par exemple au Critical Human Geography dirigé par Mark Billinge, Derek Gregory et Ron Martin et publié par Palgrave MacMillan dès 1984) et de cours à part entière dans les cursus universitaires. Depuis 1997, des rencontres annuelles sont organisées par le groupe international de géographie critique, réseau récemment constitué et où les anglophones dominent numériquement, linguistiquement et sur le plan de la production théorique ${ }^{19}$. Si la pensée critique commence à faire l'objet d'un intérêt en tant que tel (voir par exemple le séminaire sur les «pensées critiques » contemporaines organisé par Séverine Chauvel à l'EHESS au printemps 2012) les géographies critiques françaises s'épanouissent plus librement, dans le cadre de laboratoires de recherche qui ne se revendiquent pas comme critiques même quand ils sont dotés de fortes traditions en la matière (en géographie sociale, en géographie des mobilités, en géographie politique, ou en environnement...). La géographie sociale se considère certes parfois comme l'équivalent, en France, des approches critiques anglophones (selon Nicolas Bautès et Clément Marie dit Chirot). Mais cette absence d'organisation en chapelles explique (parmi d'autres raisons) le manque de notoriété des travaux menés en France par rapport aux approches anglophones et leur faible visibilité en tant que champ, ce qui favorise peu, pour l'instant, des rapprochements autres que thématiques.

\footnotetext{
${ }^{17}$ Les encouragements à l'engagement « communautaire » commencent à se développer au sein des universités, mais du côté des étudiants à qui sont parfois offerts des possibilités de validation d'unité d'enseignement. L'université Paris Diderot propose ainsi à ses étudiants de valider des UE «d'engagement » « citoyen et/ou solidaire + bénévole + laïque » plus caritatif cependant que militant ou politique.

${ }^{18}$ Cependant plus ouverte à l'international et moins radicalement anglophone qu' Antipode.

${ }^{19}$ Voir, dans ce numéro, le compte-rendu, par Cécile Gintrac, Martine Drozdz et Sarah Mekdjian, de la $6{ }^{\mathrm{e}}$ conférence internationale de géographie critique qui s'est tenue à Francfort du 16 au 20 août 2011.
} 
Du fait de la pluralité des approches critiques « à la française », ce numéro ne saurait prétendre à l'exhaustivité. Les textes réunis ici sont nécessairement le fruit du hasard et des disponibilités individuelles des chercheurs qui nous ont fait l'honneur de porter quelque intérêt à notre projet. Il s'agit donc modestement d'illustrer quelques-unes des manières dont des géographes travaillant dans le contexte institutionnel français (plus que la géographie ou même les « géographies françaises ») participent, avec les outils, les concepts et les héritages de leur discipline, au mouvement de renouveau des pensées critiques dans les sciences humaines et sociales. La piste pointée par Nicolas Bautès et Clément Marie dit Chirot d'une réflexion sur la place des pensées critiques dans l'enseignement de la géographie en France et de la production d'une pensée critique dans le cadre des pratiques d'enseignement nous semble de ce point de vue très fructueuse.

\section{Bibliographie}

Boltanski L. (1990) «Sociologie critique et sociologie de la critique », Politix, $\mathrm{n}^{\circ}$ 10-11, pp. 124-134.

Brennetot A. (2009) Geoéthique des territoires. Le débat public territorial à travers la presse magazine d'opinion en France, Thèse de doctorat en Géographie, Université de Rouen. URL : http://tel.archives-ouvertes.fr/tel-00592087

Brennetot A. (2011) «Les Géographes et la justice spatiale. Généalogie d'une relation compliquée », Annales de Géographie, $\mathrm{n}^{\circ}$ 2, pp. 115-134.

Brun J. (1994) «Essai critique sur la notion de ségrégation et sur son usage en géographie urbaine », in Brun J., Rhein C. (dir.) La ségrégation dans la ville, Paris, L'Harmattan, pp. 2157.

Calbérac Y. (2010) Terrains de géographes, géographes de terrain. Communauté et imaginaire disciplinaires au miroir des pratiques de terrain des géographes français du $\mathrm{XX}^{\mathrm{e}}$ siècle. Thèse de doctorat en Géographie, Université Lumière Lyon 2. URL: http://tel.archivesouvertes.fr/tel-00551481

Calbérac Y. et Delage A. (2010) «Introduction. L'approche spatiale comme moyen de compréhension et d'action sur les sociétés ». Dossier «A quoi sert la géographie? » Tracés, revue de sciences humaines, hors-série, pp. 121-134.

Collectif d'auteurs (2011) Penser à gauche. Figures de la pensée critique aujourd'hui, Paris, Editions Amsterdam, 505 p.

Collignon B. (2001) «La géographie radicale à la recherche d'un nouveau souffle. Introduction », in Staszak J-F. (dir.), Géographies anglo-saxonnes. Tendances contemporaines, Paris, Belin, pp. 131-138. 
Collignon B. (2010) «L'éthique et le terrain », L’information géographique, 74, 1, pp. 63-83.

Cusset (2003) French Theory. Foucault, Derrida, Deleuze \& Cie et les mutations de la vie intellectuelle aux États-Unis, Paris, Éditions La Découverte, 367 p.

Cusset F. (2006) La grande décennie. Le grand cauchemar des années 1980, Paris, La Découverte, $370 \mathrm{p}$.

Cusset F. et Keucheyan R. (2010) « Nouvelles pensées critiques ;?, entretien avec T. Labica», http://www.contretemps.eu/interviews/nouvelles-pens $\% \mathrm{C} 3 \% \mathrm{~A} 9 \mathrm{es}-\mathrm{critiques}$-entretien-razmigkeucheyan-fran $\% \mathrm{C} 3 \% \mathrm{~A} 7$ ois-cusset

Dresch J. (1979) Un géographe au déclin des empires, Paris, Maspero, 261 p.

Fossier A. et Manicki A. (2007) «Où en est la critique ? », Tracés. Revue de Sciences humaines, $\mathrm{n}^{\circ} 13$, pp. ;-5-22. URL : $\underline{\text { http://traces.revues.org/306 }}$

Hage J. (2010) Feltrinelli, Maspero, Wagenbach : une nouvelle génération d'éditeurs politiques d'extrême-gauche en Europe occidentale, 1955-1982, Thèse de doctorat en Histoire, Université de Versailles - St-Quentin-en-Yvelines.

Kalinowski I. (2005) Leçons wéberiennes sur la science et la propagande, in Max Weber, La Science, profession et vocation (textes réunis et présentés par Isabelle Kalinowski), Marseille, Agone, 299 p.

Keucheyan R. (2010) Hémisphère gauche. Une cartographie des nouvelles pensées critiques, Paris, La Découverte, 316 p.

Lacoste Y. (1976) La géographie, ça sert, d'abord, à faire la guerre, Paris, Maspero, 187 p.

Lévy J. (1995) Egogéographies. Matériaux pour une biographie cognitive, Paris, L’Harmattan, $190 \mathrm{p}$.

Morelle M. et Ripoll F. (2009) «Les chercheur-es face aux injustices : l'enquête de terrain comme épreuve éthique », Annales de Géographie, n 665-666, pp. 157-168.

Ory P. et Sirinelli J.-F. (1986) Les intellectuels en France de l'affaire Dreyfus à nos jours, Paris, Armand Colin, 263 p.

Rochefort R. (1961) Le travail en Sicile. Etude de géographie sociale, Paris, PUF, 364 p.

Volvey A., Calbérac Y. et Houssay-Holzschuch M. (dir.) (2012) «Terrain de je. (Du) sujet (au) géographique ». Annales de géographies, $n^{\circ} 687$.

Wacquant L. (2001) Entretien «El pensamiento crítico como disolvente de la doxa », Adef : Revista de Filosofía, 26-1 (Mai), 2001, (Buenos Aires), pp. 129-134, URL: http://www.homme-moderne.org/societe/socio/wacquant/pensecri.html

Wiggerhaus R. (1993) L’École de Francfort. Histoire, développement, signification, Paris, PUF, $694 \mathrm{p}$.

Walzer M. (1990) Critique et sens commun. Essai sur la critique sociale et son interprétation, Paris, La Découverte, 111 p. 